\title{
Crunching Dilaton, Hidden Naturalness
}

\author{
Csaba Csáki, ${ }^{1}$ Raffaele Tito D’Agnolo๑, ${ }^{2}$ Michael Geller@, ${ }^{3}$ and Ameen Ismail@ ${ }^{1}$ \\ ${ }^{1}$ Department of Physics, LEPP, Cornell University, Ithaca, New York 14853, USA \\ ${ }^{2}$ Institut de Physique Théorique, Université Paris Saclay, CEA, F-91191 Gif-sur-Yvette, France \\ ${ }^{3}$ School of Physics and Astronomy, Tel-Aviv University, Tel-Aviv 69978, Israel
}

(Received 9 September 2020; revised 28 January 2021; accepted 9 February 2021; published 1 March 2021)

\begin{abstract}
We introduce a new approach to the Higgs naturalness problem. The Higgs mixes with the dilaton of a conformal field theory (CFT) sector whose true ground state has a large negative vacuum energy. If the Higgs vacuum expectation value is nonzero and below $\mathcal{O}(\mathrm{TeV})$, the CFT admits a second metastable vacuum, where the expansion history of the Universe is conventional. As a result, only Hubble patches with unnaturally small values of the Higgs mass do not immediately crunch. The main experimental prediction of this mechanism is a dilaton in the $0.1-10 \mathrm{GeV}$ range that mixes with the Higgs and can be detected at future colliders and experiments searching for weakly coupled particles.
\end{abstract}

DOI: 10.1103/PhysRevLett.126.091801

Introduction.-While the discovery of the Higgs boson at the Large Hadron Collider [1,2] marked a triumph for the standard model of particle physics, the measured values of its mass and vacuum expectation value (VEV) remain unexplained. The Higgs VEV determines the mass scales of all elementary particles in the standard model (SM) and hence greatly influences all phenomenology at the weak scale and below, including, for instance, the stability of nuclei and stars.

The traditional approaches for explaining the value of the Higgs boson mass predict new colored particles around a $\mathrm{TeV}$. These approaches are under ever-increasing experimental pressure, since the Large Hadron Collider (LHC) did not discover any evidence for physics beyond the SM at the $\mathrm{TeV}$ scale $[3,4]$. It is then natural to wonder whether there are other approaches to the problem that are not tied to the appearance of new particles at a $\mathrm{TeV}$ or, more specifically, observable "top partners." One well-known idea is neutral naturalness. In its classic implementation, twin Higgs [5], the top partners interact only very weakly with the SM and are not produced at observable rates at colliders. These models, however, generally predict deviations in Higgs coupling measurements at the LHC that have not yet been observed.

A radical departure from these symmetry-based solutions are mechanisms for cosmological selection of the Higgs mass [6-11]. In these approaches, the corrections to the Higgs potential from high energy scales are

Published by the American Physical Society under the terms of the Creative Commons Attribution 4.0 International license. Further distribution of this work must maintain attribution to the author(s) and the published article's title, journal citation, and DOI. Funded by SCOAP ${ }^{3}$. unsuppressed; the lightness of the Higgs is instead realized by the cosmological dynamics of new fields.

The most extreme break with standard beyond the standard model (BSM) lore is to rely on a fully anthropic reasoning for the existence of a light Higgs in the context of a multiverse with several patches, each corresponding to a different Higgs VEV. This approach has been advocated by [12] and is also partly the motivation behind split supersymmetry (SUSY) models [13].

We introduce a novel approach to the Higgs hierarchy problem that incorporates the most appealing elements from the three classes of solutions described above. The Higgs VEV plays a dominant role in the existence of a vacuum where the cosmological constant $(\mathrm{CC})$ can be positive, allowing for inflation and postinflationary expansion of the Universe. To this end, we introduce a spontaneously broken conformal field theory (CFT), which has a large and negative vacuum energy in its true ground state, leading to an immediate cosmic crunch. This sector is charged under the electroweak (EW) gauge group and couples to the fundamental Higgs field. If the Higgs VEV is sufficiently small-but not zero- the mixing of the Higgs with the dilaton field (setting the scale for the breaking of scale invariance) can generate a second, cosmologically long-lived vacuum with a small positive $\mathrm{CC}$. The selection of a small Higgs VEV is thus dynamical and happens during the early history of the Universe. For this to be possible, we need Higgs VEVs close to the weak scale to have an $\mathcal{O}(1)$ effect on the dilaton potential. This is naturally realized as a consequence of scale invariance, like in symmetry-based solutions to the hierarchy problem.

As in the anthropic approach, we assume the presence of a multiverse with a large number of causally disconnected Hubble patches, each having a different Higgs mass parameter. Only patches where the Higgs VEV is 
$\mathcal{O}(100 \mathrm{GeV})$ survive crunching and undergo expansion, leading to a dynamical selection of regions with small Higgs VEV that appear unnatural to a low energy observer. In contrast to the regular anthropic approach, where the Higgs is typically much heavier in most of the Universe, here EW-scale masses dominate the Universe on cosmological timescales.

Our mechanism makes several experimental predictions for current and future colliders. First, we expect KK partners of the Higgs and electroweak gauge bosons to lie close to the EW scale, but we do not predict any light top partners. In fact, our EW Kaluza-Klein (KK) states have nothing to do with the cancellation of quadratic contributions to the Higgs mass. Current LHC bounds on such states stretch to the $\mathrm{TeV}$ range, generating a "little hierarchy" in our model.

Second, due to this little hierarchy, the dilaton must be light, below $\sim 10 \mathrm{GeV}$ - testable in rare $B$ decays, searches for weakly coupled light particles, future precision $Z$ experiments, and heavy-ion collisions. UV completions of the model that can generate the little hierarchy without fine-tuning will likely require a form of supersymmetry that results in a split SUSY-like spectrum.

The basic concept.-We assume a landscape of Higgs mass and cosmological constant values with a cutoff at the scale $\Lambda$

$$
V_{H}(H)=-m_{H, i}^{2} H^{\dagger} H+\lambda\left(H^{\dagger} H\right)^{2},
$$

where a typical $m_{H, i}^{2}$ is $\mathcal{O}\left(\Lambda^{2}\right)$. We remain agnostic as to how this landscape is generated and populated.

We introduce dynamics that can support the expansion of the Universe only when the Higgs VEV, $h \equiv\left\langle H^{0}\right\rangle$, is in a finite range

$$
H_{I} \lesssim h_{\min } \lesssim h \leq h_{\text {crit }} \simeq \mathcal{O}(1 \mathrm{TeV})
$$

and cause an immediate crunch for other values. In the above equation, $H_{I}$ is Hubble during inflation. Such dynamics excludes all positive and large negative mass terms for the Higgs, and only values of the VEV below the weak scale survive inflation. The mechanism is not sensitive to the minimal value $h_{\min }$, which can be generated in many ways, briefly discussed in the Cosmological Constraints section.

The dynamics needed to achieve this is based on the mixing of the Higgs with a spontaneously broken CFT—or a bulk Higgs in the anti-de Sitter (AdS) picture with the Higgs potential, Eq. (1), on the UV brane. The CFT is spontaneously broken via the Goldberger-Wise (GW) mechanism, with the GW minimum for the dilaton $\langle\chi\rangle=$ $\chi_{\mathrm{GW}}$ at a value of the potential above $\Lambda^{4}$ and the scale of inflation $M_{I}^{4} \simeq M_{\mathrm{Pl}}^{2} H_{I}^{2}$, so that the total vacuum energy in this minimum is always negative, even during the slow-roll regime of inflation. Any patch in which the dilaton has reached this minimum crunches. The heart of our mechanism is the generation of a second minimum for the dilaton by the bulk Higgs VEV, for which the vacuum energy is subdominant to the inflaton vacuum energy; any patch in this second minimum then goes through inflation without crunching. This minimum only exists for a finite range of small Higgs VEVs, set by the parameters of the bulk Higgs. Therefore, only this range of VEVs survive after inflation and until today. These small values are not typical in the landscape, thus generating a hierarchy and an apparent naturalness problem. We assume that one of the usual mechanisms (such as scanning in the multiverse plus anthropic selection) ensures a small positive $\mathrm{CC}$ in the shallower metastable minimum, while it cannot overcome the large negative energy of the true minimum.

RS model.-We use the 5D warped description [14] of the CFT with the Higgs field in the bulk of AdS space

$$
d s^{2}=\left(\frac{R}{z}\right)^{2}\left(\eta_{\mu \nu} d x^{\mu} d x^{\nu}-d z^{2}\right)
$$

Here $R=1 / k$ is the AdS curvature and the location of the UV brane, while $R^{\prime}$ is the location of the IR brane, with $\chi=1 / R^{\prime}$ identified with the dilaton-radion field [15-17]. Note that the dilaton defined this way is not canonically normalized-its kinetic term is $3\left(N^{2}-1\right) / 4 \pi^{2}\left(\partial_{\mu} \chi\right)^{2}$, where $N$ is the number of colors in the dual CFT picture, related to the 5D parameters by $N^{2}-1=16 \pi^{2}\left(M_{*} R\right)^{3}$, where $M_{*}$ is the $5 \mathrm{D}$ Planck scale.

The GW stabilization field [18] gives rise to the usual GW potential for the dilaton [17] that we parametrize as

$$
V_{\mathrm{GW}}(\chi)=-\lambda \chi^{4}+\lambda_{\mathrm{GW}} \frac{\chi^{4+\delta}}{k^{\delta}}
$$

We imagine that the GW minimum is at large negative values of the potential, such that the negative CC generated when $\chi$ is at this minimum is larger than any other scale in the theory and leads to a rapid cosmic crunch.

The novel pieces of the effective potential (valid below the warped-down local cutoff $\Lambda_{\mathrm{IR}}$ ) necessary for generating the Higgs-dependent second minimum are

$V_{H \chi}(\chi, H)=\lambda_{2}|H|^{2} \frac{\chi^{2+\alpha}}{k^{\alpha}}-\lambda_{H \epsilon}|H|^{2} \frac{\chi^{2+\alpha+\epsilon}}{k^{\alpha+\epsilon}}-\lambda_{4}|H|^{4} \frac{\chi^{2 \alpha}}{k^{2 \alpha}}$,

and we assume that the couplings $\lambda, \lambda_{\mathrm{GW}}, \lambda_{2}, \lambda_{\mathrm{H}}$, and $\lambda_{4}$ are all positive. These additional terms can be easily generated from interactions localized on the IR brane. For a detailed discussion of the origin of these terms in the 5D picture and discussion of the UV vs IR cutoffs, as well as the CFT interpretation of our scheme, we refer the reader to the Supplemental Material [19], which includes Refs. [20-27]. 


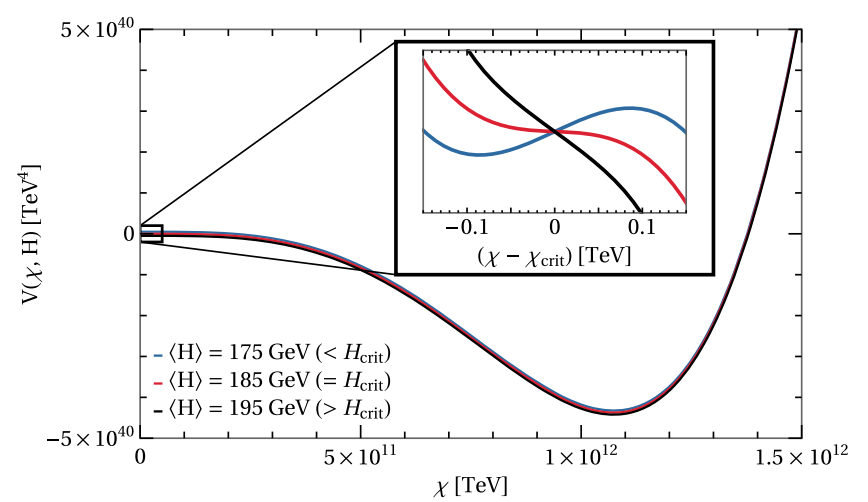

FIG. 1. The dilaton potential $V(\chi, H)=V_{\mathrm{GW}}(\chi)+V_{H \chi}(\chi, H)$ for three different values of the Higgs VEV $\langle H\rangle$, using $k=10^{8} \mathrm{TeV}, \quad \lambda_{\mathrm{GW}}=1.2 \times 10^{-5}, \quad \lambda=1.1 \lambda_{\mathrm{GW}}, \quad \lambda_{2}=0.005$, $\lambda_{H, \epsilon}=0.018, \lambda_{4}=3, \delta=0.01, \alpha=0.05$, and $\epsilon=0.1$. The true vacuum is depicted in the main figure, while the second minimum is visible in the inset; note the potentials are shifted so that the inflection point $\chi_{\text {crit }}$ lies at the origin.

Dynamics of the dilaton-Higgs potential.-Let us now investigate the dynamics resulting from the Higgs-dilaton potential $V(\chi, H)=V_{\mathrm{GW}}(\chi)+V_{H \chi}(\chi, H)+V_{H}(H)$, where $V_{\mathrm{GW}}$ and $V_{H \chi}$ are given in Eqs. (4) and (5) and $V_{H}$ is the UV brane-localized SM Higgs potential in Eq. (1). To ensure that the Higgs mass is still dominated by $V_{H}$ we take the exponent $\alpha$ in Eq. (5) to be positive and not too large, implying $m_{b}^{2} \simeq-3$ (and hence a Higgs field linear in $z$ ). We also take $V_{\mathrm{GW}}$ to be subdominant to $V_{H \chi}$ at small values of $\chi$, around the $V_{H \chi}$ minimum.

For a finite range of Higgs values the $\chi$ potential admits two minima, one generated by $V_{\mathrm{GW}}$ and the other by $V_{H \chi}$. Above the critical value of the Higgs VEV $h_{\text {crit }}$ the latter disappears, leaving only the GW minimum (see Fig. 1). The minimum also disappears when the Higgs VEV is zero. The Higgs VEV in our part of the Universe must be smaller than $h_{\text {crit }}$ or the dilaton would have rolled down to the GW minimum, resulting in a crunch. Hence, there is no tuning associated with the Higgs VEV.

If we neglect $V_{\mathrm{GW}}$ at small $\chi, h_{\text {crit }}$ can be computed by finding the value of $h$ for which $\partial_{\chi} V_{H \chi}$ has only one zero,

$h_{\text {crit }}=k\left(\frac{\lambda_{2}}{\lambda_{H \epsilon}} \frac{4-\alpha^{2}}{(2+\epsilon)^{2}-\alpha^{2}}\right)^{\frac{1-\alpha / 2}{\epsilon}} \sqrt{\frac{\lambda_{2}}{\lambda_{4}} \frac{\epsilon(2+\alpha)}{2 \alpha(2-\alpha+\epsilon)}}$.

For $h \lesssim h_{\text {crit }}$, we can also estimate the second minimum of the $\chi$ potential as

$$
\chi_{\min } \simeq\left(\frac{h^{2}}{k^{\alpha}} \frac{2 \alpha \lambda_{4}}{(2+\alpha) \lambda_{2}}\right)^{\frac{1}{2-\alpha}}
$$

neglecting the $\lambda_{H \epsilon}$ term, which is suppressed at the minimum by $\left(\chi_{\min } / k\right)^{\epsilon}$ relative to $\lambda_{2}$. For small $\epsilon$, a mild hierarchy between couplings $\lambda_{2} \lesssim \lambda_{H \epsilon}$ can generate a large hierarchy of scales $h_{\text {crit }}, \chi_{\min } \ll k$.

$\chi_{\min }$ sets the size of the extra dimension, hence determining the mass scale of new states potentially observable at colliders. The little hierarchy problem is reflected in the need to impose a mild hierarchy $h / \chi_{\min } \lesssim 0.1$. This implies a hierarchy of couplings $\lambda_{2}, \lambda_{H \epsilon}<10^{-2} \alpha \lambda_{4}$. The resulting tuning (along with the naïve dimensional analysis for these couplings) is discussed in detail in the Supplemental Material [19].

The most interesting consequence of this little hierarchy is the prediction of a light dilaton. Its mass for small $\epsilon, \alpha$, and $\delta$ is

$$
m_{\chi} \simeq m_{h} \sqrt{\frac{h}{\chi_{\min }} \frac{\pi \sin \theta}{\sqrt{6} N}-\frac{8 \pi^{2}\left(\lambda-\lambda_{\mathrm{GW}}\right)}{N^{2}} \frac{\chi_{\min }^{2}}{m_{h}^{2}},}
$$

where

$$
\sin \theta \sim \frac{\left(\lambda_{2}-\lambda_{H \epsilon}\right)}{N} \frac{h \chi_{\min }}{m_{h}^{2}}
$$

parametrizes the dilaton mixing with the Higgs.

As stated above, the previous analysis is valid only if the GW potential is subleading to $V_{\chi H}$ around $\chi_{\text {crit }}$, leading to an upper bound $\lambda \sim \lambda_{\mathrm{GW}} \lesssim \lambda_{2}^{2} / \lambda_{4}$. If $V_{\mathrm{GW}}$ dominates over $V_{\chi H}$ at $\chi_{\text {crit }}$, it washes out the metastable minimum.

Phenomenological consequences.-While we are dealing with a warped 5D model, the essence of our mechanism for a light Higgs is completely different from a vanilla holographic composite Higgs model. Our theory does not have top partners, light or heavy; they play no role in the stabilization of the Higgs hierarchy. There are no KK gluons either. There have to be KK electroweak gauge bosons, since the Higgs propagates in the bulk, but they do not have to be light and also play no role in stabilizing the hierarchy. The Higgs gets a large fraction of its potential on the UV brane and can be thought of as a mixture of elementary and composite states.

The most salient phenomenological feature of our model is the existence of a light dilaton, as shown in Eq. (8). Because of its mixing with the Higgs it inherits all SM Higgs couplings suppressed by the mixing angle $\theta$. In addition to these, the dilaton has direct couplings to the SM fields. Since the SM fermions are assumed to be localized on the UV brane and the dilaton is localized predominantly on the IR brane, their direct couplings are negligible. In contrast, electroweak gauge bosons propagate in the bulk and their direct coupling to the dilaton is given by [16,27]

$$
\frac{\chi}{2 \chi_{\min } \log \frac{R^{\prime}}{R}}\left(F_{\mu \nu}^{2}+Z_{\mu \nu}^{2}+2 W_{\mu \nu}^{2}\right) .
$$

The direct couplings to the $Z, W$ mass terms are a small correction to those obtained from the mixing with the Higgs, and their effects can be neglected. 
One can easily obtain an upper bound on the dilaton mass $m_{\chi} \lesssim 10 \mathrm{GeV}$ from Eqs. (8) and (9). Assuming we do not tune the two terms in Eq. (8), we also expect a lower bound on $m_{\gamma}$ of $\mathcal{O}(100) \mathrm{MeV}$. These bounds are derived in full detail in the Supplemental Material [19].

In summary, we have a dilaton with mass $0.1 \lesssim m_{\chi} \lesssim$ $10 \mathrm{GeV}$ and couplings to fermions proportional to $\sin \theta \sim m_{\chi}^{2} / m_{h}^{2}$. The direct coupling to photons in Eq. (10) plays an important role in its phenomenology, giving an $\mathcal{O}(1)$ correction to its branching ratios.

To explore the properties of this dilaton and the experimental constraints on it, we randomly generated $10^{5}$ points in the parameter space, fixing the parameters $k=10^{11} \mathrm{GeV}, \delta=0.01, N=3$, and $\alpha=0.05$, while uniformly sampling the other parameters from the ranges $\lambda_{\mathrm{GW}} \in(0.5,1.5) \times 10^{-5}, \quad \lambda_{2} \in(0.5,1.5) \times 10^{-2}$, $\lambda_{H \epsilon} \in(2,4) \cdot \lambda_{2}, \lambda_{4} \in(2,3)$, and $\epsilon \in(0.03,0.1)$. We also took $\lambda=1.1 \lambda_{\mathrm{GW}}$ and set the Higgs $\operatorname{VEV}\langle H\rangle \simeq 174 \mathrm{GeV}$. The parameter values chosen here reflect the little hierarchy $h_{\text {crit }} / \chi_{\min } \lesssim 0.1$ and $V_{\mathrm{GW}} \lesssim V_{H \chi}$ at $\chi_{\min }$. This is explained in full detail in the Supplemental Material [19]. $k$ could be taken larger than $10^{11} \mathrm{GeV}$ if desired, up to the Planck scale; in this case, one can just as easily find satisfactory points in the parameter space, without substantially affecting our results. To probe lower dilaton masses, we performed a similar analysis of $5 \times 10^{4}$ points, choosing $N=8, \quad \alpha=0.1, \quad \lambda_{\mathrm{GW}}=2 \times 10^{-6}, \quad \lambda_{2} \in(0.5,1) \times 10^{-2}$, and $\epsilon \in(0.05,0.1)$, while keeping the other parameters

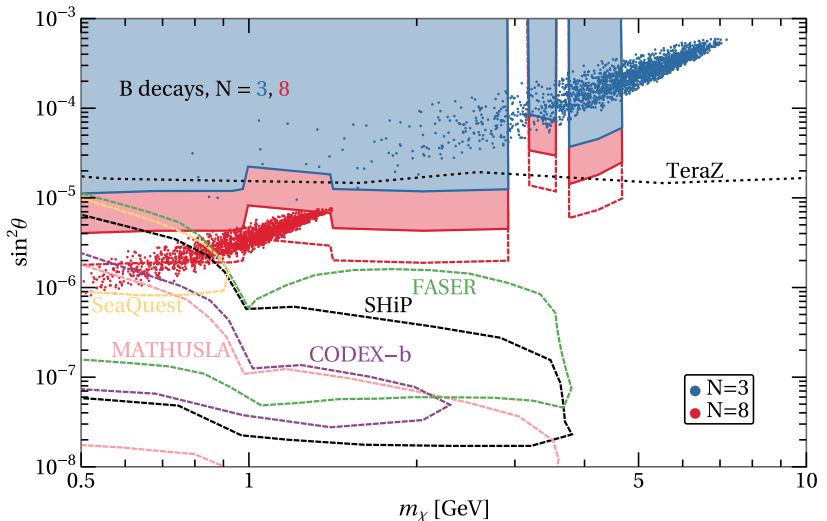

(a) the same. Points were excluded from our analysis if they failed to satisfy the following criteria: the metastable vacuum must exist and be located at $\chi_{\min }>1 \mathrm{TeV}$; $h_{\text {crit }} \leq 2 \mathrm{TeV}$ so the Higgs VEV is natural; the metastable vacuum reproduces the SM values of the Higgs mass and VEV and corresponds to a stable local minimum of the twodimensional potential; and the $\mathrm{O}(4)$ bounce action $S_{4}$ between the two potential minima is at least $\mathcal{O}(200)$ so that tunneling is suppressed.

The bounce action (see [28]) was computed by numerically solving the Euclidean equation of motion, using the shooting method to satisfy the boundary conditions. In practice, $S_{4}$ was at least $\mathcal{O}\left(10^{4}\right)$ for points satisfying the other three criteria.

The results of the two scans are plotted in Fig. 2(a). We indicate the relevant experimental bounds from rare $B$ meson decays $[29,30]$, adapted from [31,32]. The region in the $m_{\chi}-\sin \theta$ plane populated by the two scans can be understood from Eq. (8): the points approximately fall on the curve $m_{\chi} \sim \sqrt{\sin \theta}$, with upper (lower) bounds determined by the values of $\lambda_{2, H \epsilon}\left(\lambda, \lambda_{\mathrm{GW}}\right)$. The lightest $\mathrm{KK}$ Higgs and KK EW gauge bosons lie around 3-4 and 2.5-3.5 TeV, respectively, the precise number depending on the value of $\chi_{\min }$ for each point. (Note that the LHC bounds on the $\mathrm{KK} W$ and $Z$ are not significantly reduced by moving the gluons and quarks onto the UV brane, due to the tail of the KK gauge wave functions only being logarithmically suppressed on the UV brane.) There are

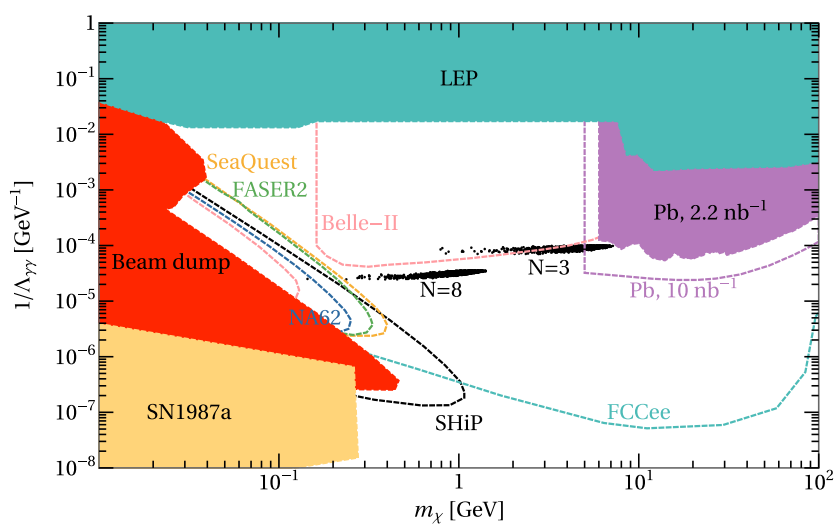

(b)

FIG. 2. (a) The dilaton mass $m_{\chi}$ and its mixing angle with the Higgs $\sin ^{2} \theta$ for randomly sampled points from our model. The blue and red points use different parameter ranges, detailed in the body of the text. We show current bounds from $B$ meson decays at LHCb [29,30] (blue and red shaded regions), adapted from [31,32]. We estimate updated $B$ decay bounds for $N=8$ following run 3 of the LHC (dotted red line), assuming an integrated luminosity of $15 \mathrm{fb}^{-1}$ [35]. We also include projections (using $N=8$ ) for bounds from searches for hidden light particles, at the beam dump experiments SHiP [33] and SeaQuest [34,35], as well as the collider experiments MATHUSLA [40,41], CODEX-b [38,39], and FASER [36,37] (dashed lines). Finally, we include a projection for $Z \rightarrow Z^{*} \chi$ at the FCCee running on the $Z$ pole (Tera- $Z$ ) [32] (dotted black line), rescaled from the corresponding LEP limits [42,43]. (b) The dilaton coupling to photons $1 / \Lambda_{\gamma \gamma}$ for randomly sampled points from our model. We include points from both of our two choices of parameter ranges. We show bounds, adapted from [45], from searches at LEP for $e^{+} e^{-} \rightarrow \gamma \chi \rightarrow 3 \gamma$ [46,47] (turquoise), beam dump experiments

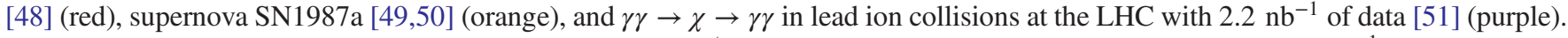

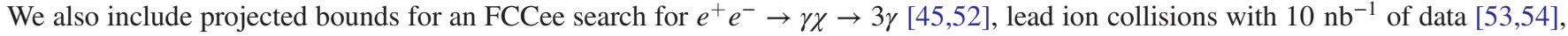
Belle-II, FASER2, NA62, SeaQuest, and SHiP (dashed lines) [55,56]. 
two regions in the parameter space free of bounds around $0.5-1.5$ and 5-7 GeV, as illustrated in Fig. 2(a) along with expected future experimental sensitivities [32-44].

Finally, in Fig. 2(b) we plot the dilaton coupling to photons $1 / \Lambda_{\gamma \gamma}$ for each point in our scans, alongside current and projected experimental bounds adapted from $[45,55,56]$. These bounds provide constraints on the model that are independent of $\sin \theta$. We normalize the coupling such that the dilaton-photon interaction term is $\left(1 / 4 \Lambda_{\gamma \gamma}\right) \tilde{\chi} F_{\mu \nu}^{2}$, with $\tilde{\chi}$ the canonically normalized dilaton. The region of parameter space populated by our model evades the existing experimental bounds [46,47,51]; projections for future sensitivities [45,52-54] are also presented in Fig. 2(b). A combination of beam dump experiments and a future lepton collider can cover all our viable parameter space.

Cosmological constraints. - First, we require that the energy density in the true vacuum is indeed always negative -i.e., $\lambda \chi_{\mathrm{GW}}^{4}>M_{I}^{4}$, which results in $k \gtrsim 17 M_{I}$ for $\lambda \simeq 10^{-5}$. In addition, if we assume that the cosmological constant problem is solved via a standard anthropic mechanism, we need to require that the highest possible CC in the landscape is below $\sim \lambda \chi_{\mathrm{GW}}^{4}$, so that all patches reaching the true minimum of the GW potential crunch. This condition may naturally arise for an $\mathcal{O}(\Lambda)$ SUSY breaking scale on the UV brane.

A second important requirement is that quantum diffusion never dominates over classical evolution of the dilaton. This prevents patches with the wrong value of $h$ to enter a phase of eternal inflation. We want to ensure that, for a Higgs VEV large enough or small enough such that the dilaton potential has only one minimum with a negative $\mathrm{CC}$, the dilaton does indeed roll to that minimum during inflation and does not get stuck in an eternally inflating phase. For large Higgs VEVs, the second derivative of the dilaton potential is always at least of $\mathcal{O}\left[v^{2} \simeq(174 \mathrm{GeV})^{2}\right]$ [see Eq. (5)], so classical rolling dominates over quantum diffusion, as we already imposed that the Hubble scale is less than the electroweak scale.

The case of a small or vanishing Higgs VEV could potentially be more problematic. Once the Higgs VEV is zero, the $\chi$ potential is a pure $\chi^{4}$ term even for very small values of $\chi$, leading to a very small second derivative near the origin. Hence for any choice of the Hubble scale during inflation there are regions that support eternal inflation. This can be avoided by a simple modification of the model following the ideas of [26,57], which was also used in [58]. We include an additional term $\lambda_{\gamma} \chi^{\gamma} \tilde{\Lambda}^{4-\gamma}$ in the $\chi$ potential, corresponding to explicit breaking of scale invariance at the scale $\tilde{\Lambda} \ll k$. This term does not change our analysis for $\chi \gg \chi_{*}$, defined as the scale where the effective quartic coupling blows up, i.e., $\chi_{*} \sim \tilde{\Lambda} \lambda_{\gamma}^{(1 / 4-\gamma)}$. However, for $\chi \lesssim \chi_{*}$ the potential is dominated by the explicit breaking term, which signals that for $\chi \lesssim \chi_{*}$ the description in terms of a dilaton breaks down. In that region, we expect the effective potential to be dominated by the mass scale $\chi_{*}$ and effectively behave as if a negative mass term of order $\chi_{*}^{2}$ was generated. Such an explicit breaking term can be generated by any relevant operator that has a negligible coupling in the UV and grows to be $\mathcal{O}(1)$ in the IR. One such realization could be to have the SM QCD (or a BSM QCD-like gauge theory) in the bulk, which we explain in detail in the Supplemental Material [19], along with the estimates for the scales necessary to achieve a successful model.

We thank Brando Bellazzini, Raman Sundrum, and Tomer Volansky for useful comments. C. C. and A. I. are supported in part by the NSF Grant No. PHY-1719877. C. C. is also supported in part by the BSF Grant No. 2016153. M. G. is supported in part by the Israel Science Foundation (Grant No. 1302/19).

[1] G. Aad et al. (ATLAS Collaboration), Phys. Lett. B 716, 1 (2012).

[2] S. Chatrchyan et al. (CMS Collaboration), Phys. Lett. B 716, 30 (2012).

[3] See, for example, https://atlas.web.cern.ch/Atlas/GROUPS/ PHYSICS/PUBNOTES/ATL-PHYS-PUB-2020-013/ and https://atlas.web.cern.ch/Atlas/GROUPS/PHYSICS/Combined SummaryPlots/EXOTICS/.

[4] See, for example, https://twiki.cern.ch/twiki/bin/view/ CMSPublic/PhysicsResultsSUS and https://twiki.cern.ch/ twiki/bin/view/CMSPublic/PhysicsResultsB2G.

[5] Z. Chacko, H.-S. Goh, and R. Harnik, Phys. Rev. Lett. 96, 231802 (2006).

[6] P. W. Graham, D. E. Kaplan, and S. Rajendran, Phys. Rev. Lett. 115, 221801 (2015).

[7] N. Arkani-Hamed, T. Cohen, R. T. D'Agnolo, A. Hook, H. D. Kim, and D. Pinner, Phys. Rev. Lett. 117, 251801 (2016).

[8] M. Geller, Y. Hochberg, and E. Kuflik, Phys. Rev. Lett. 122, 191802 (2019).

[9] C. Cheung and P. Saraswat, arXiv:1811.12390.

[10] A. Strumia and D. Teresi, Phys. Rev. D 101, 115002 (2020).

[11] G. F. Giudice, A. Kehagias, and A. Riotto, J. High Energy Phys. 10 (2019) 199.

[12] V. Agrawal, S. M. Barr, J. F. Donoghue, and D. Seckel, Phys. Rev. D 57, 5480 (1998).

[13] N. Arkani-Hamed and S. Dimopoulos, J. High Energy Phys. 06 (2005) 073.

[14] L. Randall and R. Sundrum, Phys. Rev. Lett. 83, 3370 (1999).

[15] C. Csaki, M. Graesser, L. Randall, and J. Terning, Phys. Rev. D 62, 045015 (2000).

[16] C. Csaki, M. L. Graesser, and G. D. Kribs, Phys. Rev. D 63, 065002 (2001).

[17] R. Rattazzi and A. Zaffaroni, J. High Energy Phys. 04 (2001) 021.

[18] W. D. Goldberger and M. B. Wise, Phys. Rev. Lett. 83, 4922 (1999).

[19] See Supplemental Material at http://link.aps.org/supplemental/ 10.1103/PhysRevLett.126.091801 for detailed derivation of Higgs-dilaton potential terms, the CFT interpretation of our 
model, dilaton mass bounds, tuning considerations, and the effect of including QCD in the bulk.

[20] R. S. Gupta, Z. Komargodski, G. Perez, and L. Ubaldi, J. High Energy Phys. 02 (2016) 166.

[21] N. Arkani-Hamed, S. Dimopoulos, and S. Kachru, arXiv: hep-th/0501082.

[22] M. Aaboud et al. (ATLAS Collaboration), Phys. Rev. D 98 , 052008 (2018).

[23] A. M. Sirunyan et al. (CMS Collaboration), Phys. Lett. B 798, 134952 (2019).

[24] B. Bellazzini, C. Csaki, J. Hubisz, J. Serra, and J. Terning, Eur. Phys. J. C 73, 2333 (2013).

[25] R. Sundrum, J. High Energy Phys. 01 (2011) 062.

[26] B. von Harling and G. Servant, J. High Energy Phys. 01 (2018) 159.

[27] C. Csaki, J. Hubisz, and S. J. Lee, Phys. Rev. D 76, 125015 (2007).

[28] S. R. Coleman, Phys. Rev. D 15, 2929 (1977); 16, 1248(E) (1977).

[29] R. Aaij et al. (LHCb Collaboration), J. High Energy Phys. 02 (2013) 105.

[30] R. Aaij et al. (LHCb Collaboration), Phys. Rev. Lett. 115, 161802 (2015).

[31] T. Flacke, C. Frugiuele, E. Fuchs, R. S. Gupta, and G. Perez, J. High Energy Phys. 06 (2017) 050.

[32] C. Frugiuele, E. Fuchs, G. Perez, and M. Schlaffer, J. High Energy Phys. 10 (2018) 151.

[33] S. Alekhin et al., Rep. Prog. Phys. 79, 124201 (2016).

[34] S. Gardner, R. J. Holt, and A. S. Tadepalli, Phys. Rev. D 93, 115015 (2016).

[35] J. Beacham et al., J. Phys. G 47, 010501 (2020).

[36] J. L. Feng, I. Galon, F. Kling, and S. Trojanowski, Phys. Rev. D 97, 035001 (2018).

[37] J. L. Feng, I. Galon, F. Kling, and S. Trojanowski, Phys. Rev. D 97, 055034 (2018).

[38] V. V. Gligorov, S. Knapen, M. Papucci, and D. J. Robinson, Phys. Rev. D 97, 015023 (2018).

[39] J. A. Evans, S. Gori, and J. Shelton, J. High Energy Phys. 02 (2018) 100.
[40] J. P. Chou, D. Curtin, and H. Lubatti, Phys. Lett. B 767, 29 (2017).

[41] J. A. Evans, Phys. Rev. D 97, 055046 (2018).

[42] M. Acciarri et al. (L3 Collaboration), Phys. Lett. B 385, 454 (1996).

[43] S. Schael et al. (ALEPH, DELPHI, L3, OPAL Collaborations, LEP Working Group for Higgs Boson Searches), Eur. Phys. J. C 47, 547 (2006).

[44] A. Abada et al. (FCC Collaboration), Eur. Phys. J. Special Topics 228, 261 (2019).

[45] M. Bauer, M. Heiles, M. Neubert, and A. Thamm, Eur. Phys. J. C 79, 74 (2019).

[46] K. Mimasu and V. Sanz, J. High Energy Phys. 06 (2015) 173.

[47] J. Jaeckel and M. Spannowsky, Phys. Lett. B 753, 482 (2016).

[48] J. D. Bjorken, S. Ecklund, W. R. Nelson, A. Abashian, C. Church, B. Lu, L. W. Mo, T. A. Nunamaker, and P. Rassmann, Phys. Rev. D 38, 3375 (1988).

[49] A. Payez, C. Evoli, T. Fischer, M. Giannotti, A. Mirizzi, and A. Ringwald, J. Cosmol. Astropart. Phys. 02 (2015) 006.

[50] J. Jaeckel, P. C. Malta, and J. Redondo, Phys. Rev. D 98, 055032 (2018).

[51] G. Aad et al. (ATLAS Collaboration), arXiv:2008.05355.

[52] M. Bauer, M. Neubert, and A. Thamm, J. High Energy Phys. 12 (2017) 044.

[53] S. Knapen, T. Lin, H. K. Lou, and T. Melia, Phys. Rev. Lett. 118, 171801 (2017).

[54] S. Knapen, T. Lin, H. K. Lou, and T. Melia, CERN Proc. 1, 65 (2018).

[55] D. Aloni, C. Fanelli, Y. Soreq, and M. Williams, Phys. Rev. Lett. 123, 071801 (2019).

[56] M. J. Dolan, T. Ferber, C. Hearty, F. Kahlhoefer, and K. Schmidt-Hoberg, J. High Energy Phys. 12 (2017) 094.

[57] P. Baratella, A. Pomarol, and F. Rompineve, J. High Energy Phys. 03 (2019) 100.

[58] I. M. Bloch, C. Csáki, M. Geller, and T. Volansky, J. High Energy Phys. 12 (2020) 191. 